\title{
Bach1 Modulates Heme Oxygenase-1 Expression in the Neonatal Mouse Lung
}

\author{
SACHA KASSOVSKA-BRATINOVA, GUANG YANG, KAZUHIKO IGARASHI, AND PHYLLIS A. DENNERY \\ Division of Neonatology [S.K.-B., G.Y., P.A.D.], School of Medicine [P.A.D.], University of Pennsylvania, Philadelphia, Pennsylvania \\ 19104; Department of Biochemistry [K.I.], Tohoku University School of Medicine, Sendai 980-8575, Japan
}

\begin{abstract}
Heme oxygenase-1 (HO-1), the rate-limiting enzyme of heme degradation and antioxidant defense protein, is induced in the lungs of animals exposed to hyperoxia. However, high levels of HO-1 expression may be deleterious, thus necessitating tight regulation. Previous reports show maturational differences in rat HO-1 regulation in hyperoxia, as newborns do not up-regulate HO-1mRNA compared with adults. To better understand the differential response of lung HO-1 to hyperoxia, we exposed newborn and adult mice to $>95 \%$ oxygen. The newborn lungs had reduced HO-1 mRNA induction compared with adults and newborn transgenic mice overexpressing luciferase driven by the $15 \mathrm{~kb} \mathrm{HO}-1$ promoter (HO-1/Luc $\mathrm{Tg}$ ) had less increased light emission in hyperoxia compared with adults. Compared with adults, levels of the repressor of HO-1 transcription, Bach1, were higher in the neonatal lung as was nuclear protein-DNA binding to the antioxidant response element (ARE) from HO-1. Furthermore, at baseline and in hyperoxia, chromatin immunoprecipitation (ChIP) revealed increased Bach1 binding to the HO-1 distal enhancers (DEs) in the neonates compared with adults. These data suggest that elevated levels of Bach1 may help to limit HO-1 induction in the newborn at baseline and in response to oxidative stress. (Pediatr Res 65: 145-149, 2009)
\end{abstract}

$\mathrm{H}$ eme oxygenase-1 (HO-1) controls the rate-limiting step in the degradation of heme to biliverdin, which in turn is rapidly converted to bilirubin via biliverdin reductase. The enzyme exists in two isoforms. HO-2 is constitutively expressed, whereas HO-1 is readily inducible in oxidative stress, such as hyperoxia (1), hypoxia (2), heavy metals (3), and ultravioletA (UVA) radiation (4) among others. It is also inducible in mammalian cells by various proinflammatory stimulants, such as cytokines and heat shock (5). The physiologic importance of HO-1 is emphasized by the phenotype of HO-1 null mice, which have a shorter life span and reduced stress defenses (6). Nonetheless, overexpression of $\mathrm{HO}-1$ has protective effects within a certain threshold (7) above which, HO-1 may be detrimental. Therefore, its expression must be tightly regulated.

The induction of HO-1 by various stressors is mainly regulated at the transcriptional level. Two distal enhancers

Received March 27, 2008; accepted September 12, 2008.

Correspondence: Phyllis A. Dennery, M.D., Division of Neonatology, The Children's Hospital of Philadelphia, 34th Street and Civic Center Boulevard, ARC Rm 415G, Philadelphia, PA 19104; e-mail: dennery@email.chop.edu

This work was supported by RO1 grant HL058752 from the National Institutes of Health (P.A.D.).
(DEs), DE1 and DE2, (8-10) upstream of the coding sequence regulate HO-1 transcription (11). Both enhancers regions contain multiple stress responsive elements (12) that also conform to the sequence of the Maf recognition element (MARE) (13). Heterodimers of basic leucine zipper factors (bZip), including NF-E2, Nrf1, Nrf2, and Nrf3, and small Maf proteins (MafK, MafF, and MafG) induce HO-1 through these MAREs (1416). MAREs are also recognized by the activator protein-1 (AP-1) family of proteins (12). Bach1, a leucine zipper protein, has been identified as a repressor of HO-1 transcription (17). In the absence of heme, it heterodimerizes with MafG or MafK proteins and binds to MAREs within the enhancer regions (18). Upon exposure to heme (19), Bach1 dissociates from its partners and is exported out of the nucleus (20). Displacement of Bach1 leads to Nrf2 recruitment to the same regulatory elements and activation of transcription $(18,21)$. Bach1 activity is also modulated by tin mesoporphyrin (22), hypoxia (23), and diamide (24). The HO-1 promoter and enhancers are situated in a permissive chromatin environment, where histone-H3 is hyperacetylated irrespective of gene activity (18). Therefore, under normal conditions in tissue culture, the chromatin structure of $\mathrm{HO}-1$ is in a preactivation state, but transcription is repressed by Bach1 $(17,18)$.

In the newborn, HO-1 is differentially regulated in hyperoxia compared with the adult (25). Despite increased HO-1 expression in the perinatal period, there was no difference in lung HO-1 mRNA levels in newly born rats exposed to hyperoxia, compared with air-exposed controls, in contrast with adult models (26). Differences in HO-1 gene expression in the newborn may be partially due to a decreased binding of AP-1 (27), but Bach1 may be involved as well. Because neonates have a relative resistance to hyperoxic regulation of $\mathrm{HO}-1$, and because Bach1 is a known negative regulator of HO-1, we wanted to understand whether this maturational difference was due to differences in lung Bach1 expression between adults and neonates.

\section{MATERIALS AND METHODS}

Animals. Wild type FVB (Charles River, Wilmington, MA) and transgenic mice, expressing the luciferase reporter gene under the control of $15 \mathrm{~kb}$ from

Abbreviations: ChIP, chromatin immunoprecipitation; EMSA, electrophoretic mobility shift assay; HO-1, heme oxygenase-1 
the mouse HO-1 promoter region and upstream regulatory sequences (HO-1/ Luc Tg; gift from Dr. Christopher H. Contag, Stanford University, Stanford, CA) were used. Newborns $(<12 \mathrm{~h})$, together with their lactating mothers, and adult (2-mo old) male mice were exposed to $>95 \% \mathrm{O}_{2}$ in a Plexiglas chamber, using a $100 \%$ oxygen cylinder in a flow system over a period of $72 \mathrm{~h}$. The oxygen levels in the chambers were monitored with an oxygen sensor. Age-matched control animals were exposed to room air. Pups were crossnurtured every day to obviate oxygen toxicity in the mothers. Animal protocols were reviewed and approved by the Institutional Animal Care and Use Committee of the Stokes Research Institute at the Children's Hospital of Philadelphia.

Tissue collection. Lungs were flushed with phosphate buffered saline (PBS) through the right ventricle, to remove blood, and they were collected and snap frozen for further studies.

In Vivo imaging of bioluminescent signal. Photon emission as a surrogate for HO-1 promoter activity was visualized in the HO-1/ Luc Tg mice using an In Vivo Imaging System [(IVIS); Xenogen, San Francisco, CA] as previously described (28). The pups were immobilized with adhesive tape. Adults were anesthetized with $2.5 \%$ isoflurane. Luciferin, the substrate for luciferase, was injected intraperitoneally ( $150 \mathrm{mg} / \mathrm{kg}$ body weight) $5 \mathrm{~min}$ before imaging. The light emitted from the HO-1/Luc $\mathrm{Tg}$ animals was detected with the IVIS. To allow for quantification and normalization, photon counts were determined from identical areas of the lungs in each animal using Living Image software (Xenogen, CA).

Quantitative real-time $\boldsymbol{R}$ T-PCR. HO-1 mRNA levels were assessed by quantitative real-time RT-PCR (qRT-PCR). Total RNA was isolated with Trizol (Invitrogen, Carlsbad, CA). First strand cDNA was synthetized with SuperscriptII Reverse transcriptase (Invitrogen, Carlsbad, CA) and random primers. Serial dilutions 1:10 of the cDNAs were made and gene-specific mRNA levels were determined with TaqMan Gene Expression Assays (900 $\mathrm{nM}$ of each primer, and $250 \mathrm{nM}$ probe (ABI) designed over exon-exon boundaries. One of the samples was used to prepare a standard curve. The mRNA levels of the samples were determined using the relative standard curve method. All reactions were performed in 384-well plates with a final volume of $10 \mu \mathrm{L}$. Real-time PCR plates were analyzed using the ABI Prism 7900HT Sequence Detection System with ABI Prism SDS2.1 software (Applied Biosystems).

Detection of HO-1 mRNA levels by Real Time PCR was performed with the Taqman Gene Expression Assay according to the manufacturer's instructions (\# Mm 00516004_m1, Applied Biosystems, Foster City, CA). The data were normalized using 18S mRNA values determined with Taqman Gene Expression Assay (\# Hs 99999901_s1, Applied Biosystems).

Analysis of protein expression. Nuclear and cytosolic protein extracts were isolated from mouse lungs by differential centrifugation according to the standard procedures with modifications (29). Briefly, lungs were homogenized in a buffer, containing $0.32 \mathrm{M}$ sucrose, $3 \mathrm{mM} \mathrm{CaCl}_{2}, 2 \mathrm{mM} \mathrm{Mg}$ Acetate, $0.1 \mathrm{mM}$ ethylenediaminetetraacetic acid (EDTA), $10 \mathrm{mM}$ Tris- $\mathrm{HCl}, \mathrm{pH} 8.0$, $0.5 \% \mathrm{NP}-40$, and $1 \mathrm{mM}$ DTT. Whole cell lysates were overlayed onto a buffer containing $2 \mathrm{M}$ sucrose, $5 \mathrm{mM} \mathrm{Mg}$ acetate, $0.1 \mathrm{mM}$ EDTA, $1 \mathrm{mM}$ DTT, 10 $\mathrm{mM}$ Tris- $\mathrm{HCl}, \mathrm{pH} 8.0$, and subjected to centrifugation at 15,500 rpm. Intact nuclei were pelleted and nuclear extracts were prepared using nuclear extraction reagent (NER) solution from the commercially available kit (Pierce NE-PER kit, Pierce Biotechnology, Rockford, IL). Protein concentrations were determined with a colorimetric Bio-Rad protein assay (Bio-Rad Laboratories, Hercules, CA).

Western blot analysis was performed with either $40 \mu \mathrm{g}$ of tissue homogenates or $20 \mu \mathrm{g}$ of nuclear extracts separated on a gradient gel $(4-12 \%$ NuPAGE, Invitrogen) and transferred onto Immobilon-P Transfer Membrane (Millipore Billerica, MA). Protein levels were detected using an enhanced chemiluminescence detection kit (GE healthcare, Piscataway, NJ) after overnight incubation of the membrane with the following antibodies: HO-1 Antibody (SPA-896, Stressgen; dilution 1:1000); Bach1 antibody (18), dilution 1:1000; Lamin B (C-20, Santa Cruz Biotechnology, dilution 1:1000), followed by secondary anti-rabbit or anti-goat-HRP- conjugated antibodies (Santa Cruz Biotechnology, Santa Cruz, CA; dilution 1:5000). Western blots were performed in triplicate. For detection of developmental expression of Bach1, we used a pool from three different samples.

Electrophoretic mobility shift assay (EMSA). A ${ }^{32} \mathrm{P}$-labeled double stranded oligonucleotide with the consensus sequence for MARE found on the HO-1 mouse gene at DE1 ( $5^{\prime}$-TTTTATGCTGTGTCATGGTT- $3^{\prime}$ and its antisense 5'-AACCATGACACAGCATAAAA-3') were used as probes to evaluate protein binding of nuclear extracts as described previously (27). Cold competition was performed in parallel reactions using a 100-fold excess of nonradiolabeled MARE to demonstrate specificity of binding. Supershift gel retardation was performed by incubation of the nuclear extracts with $2 \mu \mathrm{L}$ of Bach1 or Nrf2 antibody before addition of the radiolabeled probe. Supershift retarded bands were visualized on $\mathrm{x}$-ray film.

Chromatin immunoprecipitation. Protein binding to the HO-1 regulatory genomic region was assessed by a chromatin immunoprecipitation (ChIP) assay, according to the manufacturer's instructions (Upstate, Lake Placid, NY). Briefly, lung tissue was homogenized and DNA-associated proteins were dual cross-linked in 1\% Formaldehyde/2.5 mM EGS (Ethylene Glycolbis, Sigma Chemical) in PBS with protease inhibitors (Sigma Chemical) as described in Ref. 30. Nuclei were isolated and chromatin was prepared and sheared subsequently by a pulsed ultrasonication. Sheared DNA/protein complexes were incubated with anti Bach1 antibodies or rabbit IgG overnight. Antibody-precipitated DNA-protein complexes were reverse-crosslinked, followed by phenol/chloroform extraction, and the precipitated DNA was used as a template for PCR amplification. The occupancy of the HO-1 promoter region was assessed relative to the input on ethidium bromide stained $2 \%$ agarose gel. The DE1 region of HO-1 was amplified with primers 5'TGAAGTTAAAGCCGTTCCGG and 3'AGCGGCTGGAATGCTGAGT; DE2 region was amplified with primers 5'GGGCTAGCATGCGAAGTGAG and $3^{\prime}$ AGACTCCGCCCTAAGGGTTC.

Densitometric analysis. Intensity of signal for the immunoreactive proteins and DNA bands on ethidium bromide stained agarose gels was quantitated by densitometry using Quantity One, Bio-Rad.

Statistical analysis. Values represent the means \pm SD. Experiments were done in triplicates. For comparison between treatment groups, the Null hypothesis that there is no difference between treatment means was tested by a single factor analysis of variance (ANOVA) for multiple groups or unpaired $t$ test for two groups (Instat 3, GraphPad Software, San Diego, CA). Statistical significance $(p \leq 0.05)$ between and within groups was determined by means of the Fisher method of multiple comparisons.

\section{RESULTS}

Newborns exposed to hyperoxia have a smaller increase in HO-1 mRNA steady-state levels than adults. Newborn FVB mice had significantly higher steady-state HO-1 mRNA levels at birth compared with adults as determined by Real Time PCR and with visualization of light emission in the HO-1/Luc $\mathrm{Tg}$ mice followed from D0 to adulthood (see Fig. 1A). Thereafter, HO-1 mRNA decreased to reach adult levels (data not shown), as previously reported in neonatal rats (31). In hyperoxia, both newborns and adults showed induction of HO-1 mRNA at 24, 48, and $72 \mathrm{~h}$ of exposure, compared with the age-matched air controls (Fig. 1C). However, when comparing the fold induction between newborns and adults, the newborns showed a 2 -fold increase at 24 and $48 \mathrm{~h}$ and a 3.5 -fold increase at $72 \mathrm{~h}$ over the age-matched air-control animals (Fig. 1C) whereas adults demonstrated a significant time-dependent induction of HO-1 mRNA at each time point of hyperoxic exposure and reached a maximum of 7.9-fold by $72 \mathrm{~h}$ compared with air-exposed controls (Fig. 1C). These data demonstrate a decreased inducibility of lung HO-1 mRNA in neonates.

Lack of activation of $\mathrm{HO}-1$ promoter in the newborn mice exposed to hyperoxia. We measured the changes in HO-1 promoter activity in newborns and in adults in vivo in real time by evaluating photon emission, using $\mathrm{HO}-1 / \mathrm{Luc} \mathrm{Tg}$ mice. Basal luciferase activity was approximately 10 -fold higher in the neonates ( $<12 \mathrm{~h}$ old) (Fig. $2 A)$, compared with the adult. After $72 \mathrm{~h}$ of hyperoxia, there was moderate increase (1.5fold) in photon emission expressed as a ratio to the value obtained in age-matched controls exposed to air. These data paralleled the HO-1 mRNA levels as determined by qRTPCR. In contrast to the neonates, adults showed a 4-fold increase in photon emission after $72 \mathrm{~h}$ of hyperoxia (Fig. 2A), consistent with the increased HO-1 mRNA levels as determined by qRT-PCR.

Increased Bach1 protein levels in mouse lung in development and in hyperoxia. Because Bach1 is a known negative regulator of HO-1 transcription, we wondered whether matu- 


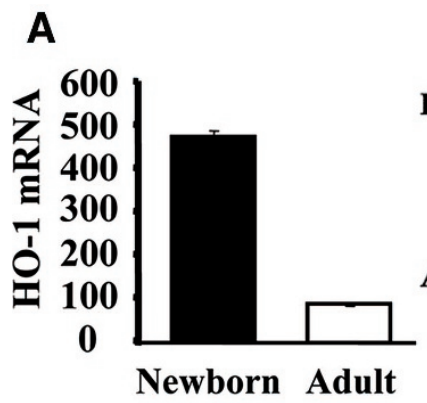

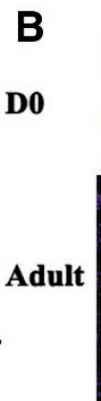

\#1

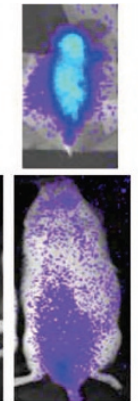

\#2

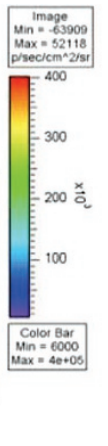

C

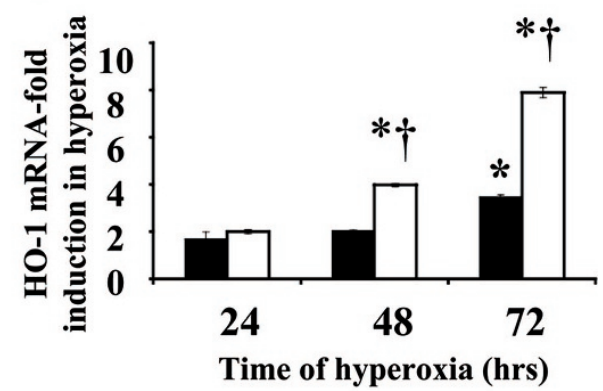

Figure 1. Basal HO-1 mRNA levels $(A)$ and basal activity of HO-1 promoter $(B)$ in neonatal mice (\#1 and \#2) followed to adulthood. HO-1 mRNA values are the ratio of HO-1 to $18 \mathrm{~S}$ mRNA. (C) Differential effects of hyperoxia on HO-1 steady-state mRNA levels in neonatal ( $\square$ ) and adult ( $\square$ ) lungs. Fold induction of HO-1mRNA compared with respective air-exposed controls is shown. ${ }^{*} p<0.05 v s .24 \mathrm{~h} ; \dagger p<0.05 v s$. similarly exposed neonates, $n=3$ for each group.

A Neonate

Air

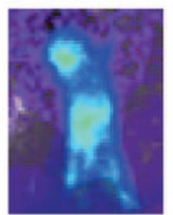

$\mathbf{O 2}$

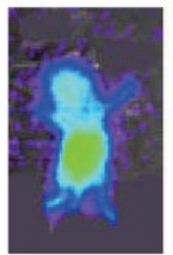

B.

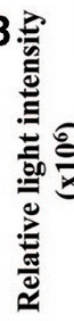

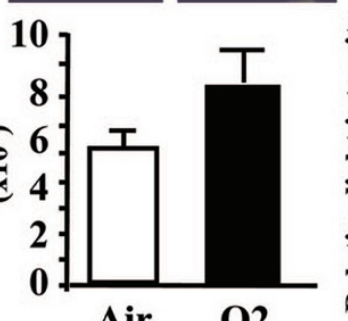

Air

02
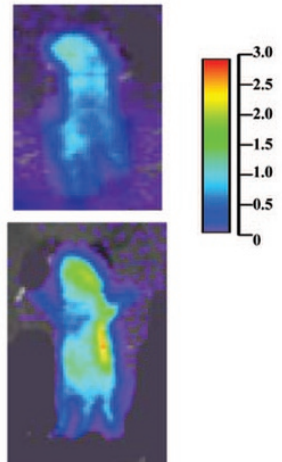
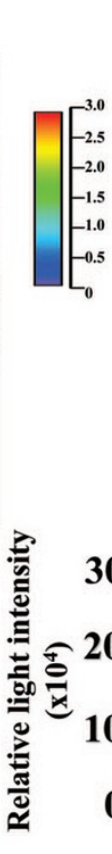

Adult
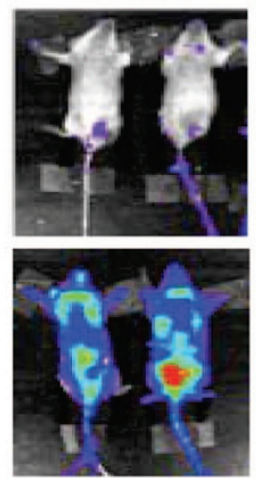

30

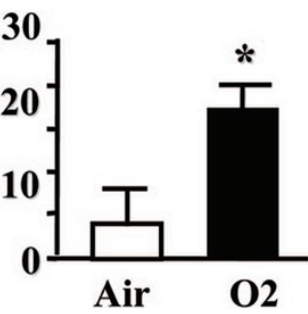

Figure 2. Visualization of HO-1 promoter activation using IVIS. (A) Representative pseudoimage of light emission as a marker of activation of HO-1 promoter in hyperoxia in neonates (left panel) and adult HO-1/Luc Tg mice (right panel) is shown. The blue to red coloring represents the lowest to highest light intensity, respectively. Animals were repeatedly imaged daily for up to $72 \mathrm{~h}$. (B) Quantitation of the light emission from the lungs of the animals in hyperoxia (ם) vs. age-matched air-controls $(\square)$. Values are the mean \pm SD of 5 measurements. $* p<0.05 v s$. air-controls.

rational differences in Bach1 could explain the relative lack of HO-1 promoter activation in hyperoxia-exposed newborn mice. Neonatal lung nuclear extracts had higher Bach1 protein levels compared with adults at baseline (Fig. 3A). Densitometry data showed highest levels of Bach1 on postnatal day 2. This peak of Bach1 was preceded by an increase of HO-1 protein at day 1 (Fig. 3A), suggesting that there is a temporal correlation between HO-1 and Bach1 such that Bach1 increases in response to HO-1. Furthermore, once Bach1 was increased on postnatal day 2, HO-1 decreased on day 3 (Fig. $3 A$ ). When animals were exposed to hyperoxia, Bach1 levels remained low in the adults, whereas in the newborns, nuclear Bach1 levels remained elevated throughout the entire hyperoxic exposure (Fig. 3B). This may explain the decreased inducibility of the neonatal lung HO-1 mRNA in hyperoxia.
A
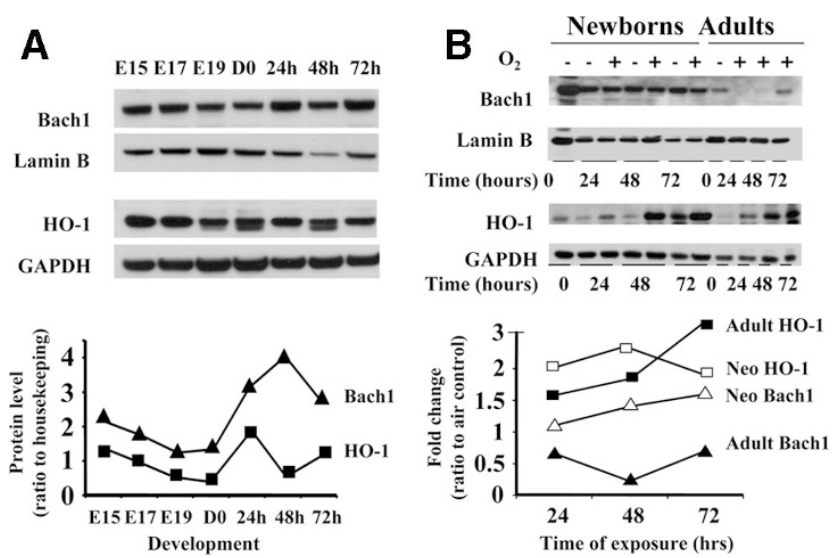

Figure 3. Ontogeny and hyperoxic regulation of Bach1 and HO-1 expression in the mouse lung. A. Bach1 and HO-1 protein levels from embryonic day E15, E17, and E19, at birth (D0), 24, 48, and $72 \mathrm{~h}$ after birth are shown. A representative Western of three pooled samples for each time point illustrates Bach1 immunoreactive signal with Lamin B as a loading control. Lung HO-1 immunoreactive signal in the same samples is also shown together with GAPDH for normalization. In the lower panel, densitometric values for Bach1 in the nucleus, normalized to Lamin B (triangles), and for HO-1 normalized to GAPDH (squares) are shown. B. A representative Western blot of nuclear Bach-1 and whole lung HO-1 immunoreactive protein in neonatal and adult animals exposed to hyperoxia are shown. Lamin B is the loading control for Bach1 and GAPDH is the loading control for HO-1. In the lower panel, densitometric values of the normalized Bach1 (triangles) and HO-1 protein levels (squares) are shown.

Lung Bach1 binding increases in newborns. After incubation of nuclear extracts from neonatal and adult lungs with radiolabeled ${ }^{32} \mathrm{P}-\mathrm{MARE}$ oligonucleotide, two major gel retardation complexes were observed. These were competed away by a cold probe, demonstrating specificity (Fig. 4). In hyperoxia, binding to MARE in the newborn nuclear lung extracts increased in a time-dependent fashion. In contrast, no timedependent increase of MARE-binding was seen in lung nuclear extracts from adult mice exposed to hyperoxia. Supershift gel retardation with Bach1 antibody further documented that Bach1 was part of the complex. Furthermore, incubation of nuclear extracts with increasing amounts of Bach1 antibody resulted in diminished intensity of the complex and increased intensity of the supershifted retarded band (Fig. 4), further confirming the specificity of binding. Interestingly, no supershift gel retardation was observed with Nrf-2 antibodies (not 


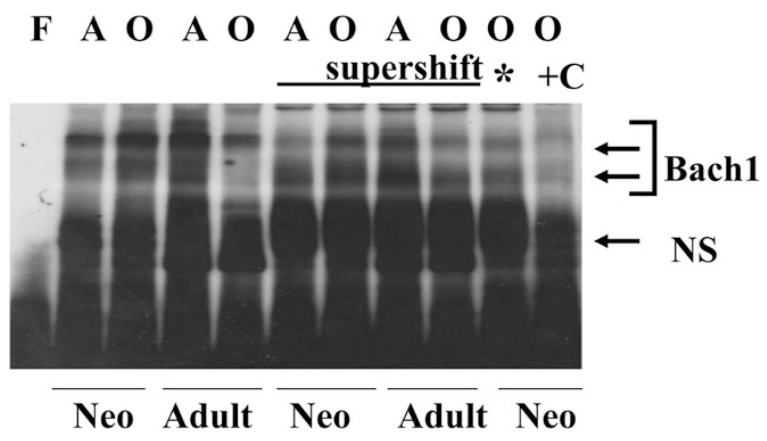

Figure 4. Increased binding of nuclear extracts from neonatal lung to the ARE. A representative of 3 EMSAs is shown. F, free probe; A, air; O, $72 \mathrm{~h}$ hyperoxia, $+\mathrm{C}$, cold competition with 100 -fold excess of unlabeled oligonucleotide probe in the nuclear extracts from hyperoxia exposed neonates. Samples were incubated with Bach1 antibodies and supershift gel retardation is shown (Supershift); The asterisk $(*)$ indicates that twice the amount of Bach1 antibody was used in the sample for a dose response; NS: Nonspecific binding. Bach1: Bach1 specific binding.

shown). Overall, higher Bach1 protein levels in the neonates corresponded with increased Bach1 binding to MARE.

Increased binding of Bach1 to HO-1 enhancers in the newborn lung. Using ChIP, lung tissues from newborns had increased binding of Bach1 antibody to the DE1 $(p=0.02$, $n=3)$ and DE2 ( $p=0.01, n=4)$ enhancers of the HO-1 promoter, compared with adults (Fig. 5A), consistent with increased Bach1 binding to MARE. In hyperoxia, Bach1 binding to the DE1 significantly increased in the newborn lung $(p<0.05)$, whereas it did not change significantly in the adult lung. When comparing the fold induction in hyperoxia versus normoxia, newborns demonstrated higher Bach1 occupancy than the adults $(2.2 \pm 0.4$ versus $1.6 \pm 0.2, p=0.036$, Fig. $5 B$ ). In hyperoxia, Bach1 binding to DE2 increased in both newborn and adult lung, but the difference between both groups was not significant $(p=0.06)$. Overall, these data suggest that enhanced DE1 binding could suppress HO-1 gene expression in neonates exposed to hyperoxia.

\section{DISCUSSION}

HO-1 is readily inducible in stress conditions such as hyperoxia, and is protective within a range of expression (32). Here, we demonstrate that neonates have increased binding of the negative regulator Bach1 compared with adults both at baseline and in hyperoxia.

We observed higher levels of HO-1 mRNA at birth in the FVB mice. This is consistent with the previously observed postnatal HO-1 mRNA expression pattern in the rat (33), (31). Additionally, in response to hyperoxia, lung HO-1 mRNA steady-state levels increased in the newborn, but less so than in the adults, suggesting decreased inducibility of HO-1 in the newborn relative to the adults. A similar trend was shown in the rat, although newborn rats had no detectable change in HO-1 mRNA after hyperoxia (25) in contrast to the FVB mice. These differences may be species-related and due to the relative susceptibility of the FVB mice to hyperoxia, as shown by others $(34,35)$. The modest level of HO-1 promoter activation in the newborn FVB mice exposed to hyperoxia is consistent with the change in HO-1 mRNA steady-state levels
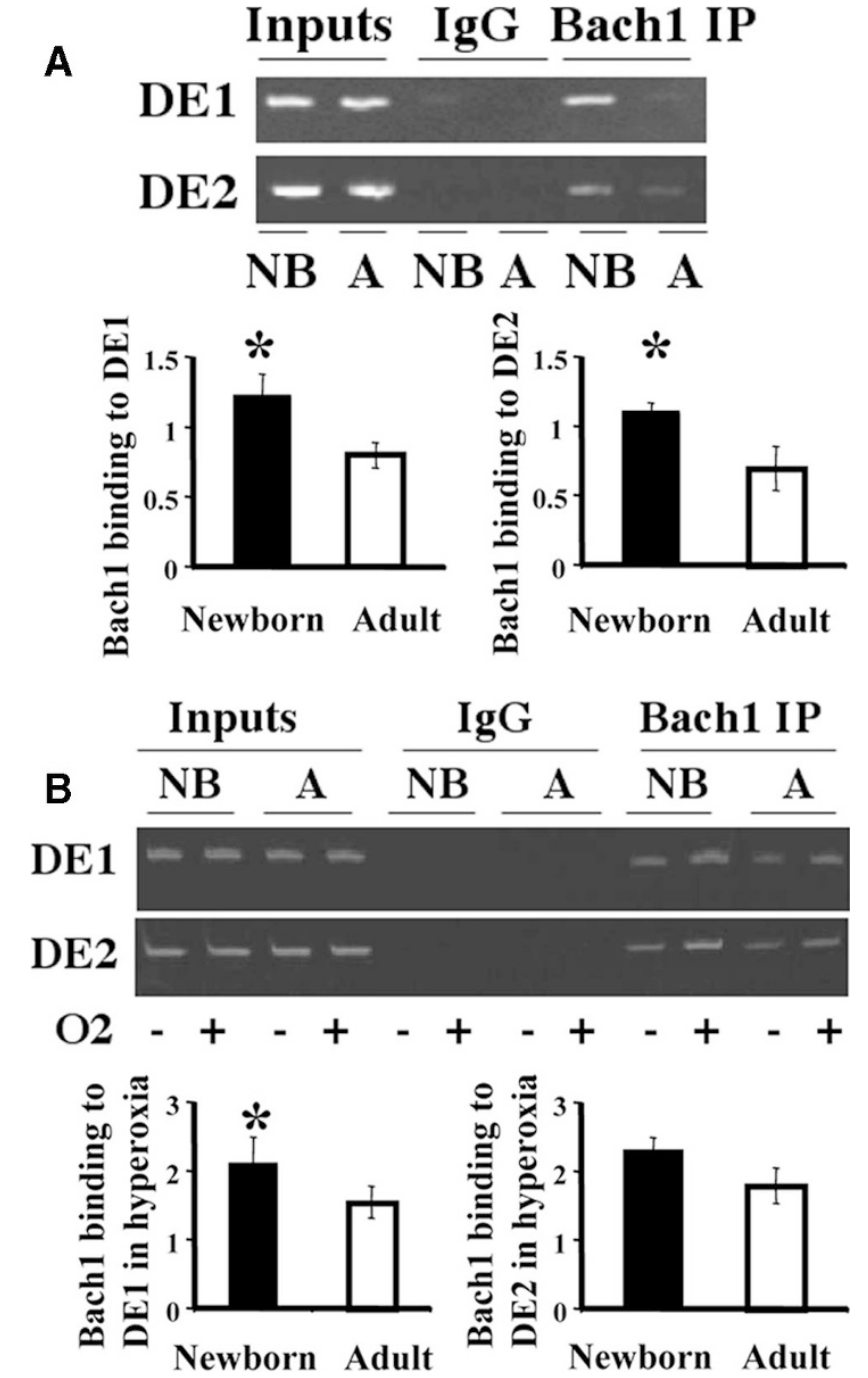

Figure 5. Increased Bach1 binding to the mouse HO-1 enhancers in neonatal mice exposed to hyperoxia. A. Bach1 binding was assessed relative to the inputs in the lungs of newborn (NB: $\square$ ) and adult (A: $\square$ ) mice at baseline. The fold change in Bach1 binding is shown. Values are mean $\pm \mathrm{SD}, p<0.05$. B. Bach1 binding was assessed relative to the inputs in the lungs of newborn and adult mice exposed for $72 \mathrm{~h}$ in hyperoxia. The fold change in Bach 1 binding $v s$. the air-matched controls is shown.

seen with qRT-PCR. The lesser induction seen with the promoter activation compared with the mRNA could be explained by posttranscriptional stabilization of HO-1 mRNA in hyperoxia in newborn FVB mice, as shown with other antioxidant enzymes $(36,37)$. This would need to be further explored. Our results demonstrate maturational differences in HO-1 hyperoxic gene regulation and a relative resistance to induction in the newborn mice.

Because Bach1 negatively regulates $\mathrm{HO}-1$, perhaps the relative resistance to hyperoxic induction of HO-1 in newborns could be explained by increased expression and binding of Bach1. We show for the first time that Bach1 has a distinct developmental profile of expression in the mouse lung. Its levels rise postnatally after the rise in HO-1 expression on postnatal day 1 . This may be an adaptive response to prevent further HO-1 induction. Bach1 heterodimerizes with small Maf proteins to bind to MARE in the HO-1 promoter. In vitro, 
HO-1 transcription is repressed by Bach1 binding $(17,18)$. Here, we show a reciprocal relationship between Bach1 and HO-1 expression during development in vivo. In contrast, in the mouse cortex, HO-1 expression parallels Bach1 expression during development (38). These differences may be due to the relative importance of Bach1 in the negative regulation of HO-1 depending on the tissue. Bach1 interactions with the chromatin remodeling machinery could also contribute to its inhibitory role, much as Nrf-2 and Brahma related gene 1 (BRG1) actively contribute to HO-1 gene expression (39). Nrf2 activity should be explored in these conditions as well.

Our data may also point to a threshold level of Bach1 needed to suppress HO-1 induction at baseline and in hyperoxia as the neonates have reduced HO-1 expression when Bach levels are at their highest whereas the adults always maintain a low level of Bach1, which may allow them to readily induce HO-1 in hyperoxia. Furthermore, the enhanced Bach1 binding to the distal elements of the HO-1 promoter in the neonates exposed to hyperoxia and the loss of this binding in adults suggests that Bach1-DNA binding inhibits HO-1 induction in neonates exposed to hyperoxia.

In summary, we demonstrated that newborn mice have increased lung Bach1 levels, nuclear protein binding to the antioxidant response element (ARE), and increased binding of Bach1 to the HO-1 DEs at baseline and in hyperoxia compared with adults. We speculate that the increased lung Bach1 levels and its increased binding to the HO-1 regulatory regions in the newborn could be a compensatory response to prevent HO-1 from reaching the threshold level where it is no longer beneficial.

Acknowledgments. We thank Ping La, Clyde Wright, Maurice Hinson, Tiangang Zhuang, Sara Lin, and Linda Gonzales for productive discussions, comments on the manuscript, and their help. We are thankful to Brenda Levin for her help with the submission of the manuscript.

\section{REFERENCES}

1. Lee PJ, Camhi SL, Chin BY, Alam J, Choi AM 2000 AP-1 and STAT mediate hyperoxia-induced gene transcription of heme oxygenase-1. Am J Physiol Lung Cell Mol Physiol 279:L175-L182

2. Yang ZZ, Zou AP 2001 Transcriptional regulation of heme oxygenases by HIF1alpha in renal medullary interstitial cells. Am J Physiol Renal Physiol 281:F900F908

3. Hamamura RS, Ohyashiki JH, Kurashina R, Kobayashi C, Zhang Y, Takaku T, Ohyashiki K 2007 Induction of heme oxygenase-1 by cobalt protoporphyrin enhances the antitumour effect of bortezomib in adult T-cell leukaemia cells. Br J Cancer 97:1099-1105

4. Prawan A, Kundu JK, Surh YJ 2005 Molecular basis of heme oxygenase-1 induction: implications for chemoprevention and chemoprotection. Antioxid Redox Signal 7:1688-1703

5. Song Y, Shi Y, Ao LH, Harken AH, Meng XZ 2003 TLR4 mediates LPS-induced HO-1 expression in mouse liver: role of TNF-alpha and IL-1beta. World J Gastroenterol 9:1799-1803

6. Poss KD, Tonegawa S 1997 Reduced stress defense in heme oxygenase 1-deficient cells. Proc Natl Acad Sci USA 94:10925-10930

7. Suttner DM, Dennery PA 1999 Reversal of HO-1 related cytoprotection with increased expression is due to reactive iron. FASEB J 13:1800-1809

8. Alam J 1994 Multiple elements within the 5' distal enhancer of the mouse heme oxygenase-1 gene mediate induction by heavy metals. J Biol Chem 269:2504925056

9. Alam J, Camhi S, Choi AM 1995 Identification of a second region upstream of the mouse heme oxygenase-1 gene that functions as a basal level and inducer-dependent transcription enhancer. J Biol Chem 270:11977-11984

10. Alam J, Wicks C, Stewart D, Gong P, Touchard C, Otterbein S, Choi AM, Burow ME, Tou J 2000 Mechanism of heme oxygenase-1 gene activation by cadmium in
MCF-7 mammary epithelial cells. Role of p38 kinase and Nrf2 transcription factor. J Biol Chem 275:27694-27702

11. Alam J, Cook JL 2003 Transcriptional regulation of the heme oxygenase-1 gene via the stress response element pathway. Curr Pharm Des 9:2499-2511

12. Inamdar NM, Ahn YI, Alam J 1996 The heme-responsive element of the mouse heme oxygenase- 1 gene is an extended AP-1 binding site that resembles the recognition sequences for MAF and NF-E2 transcription factors. Biochem Biophys Res Commun 221:570-576

13. Kataoka K, Noda M, Nishizawa M 1994 Maf nuclear oncoprotein recognizes sequences related to an AP-1 site and forms heterodimers with both Fos and Jun. Mol Cell Biol 14:700-712

14. Kataoka K, Handa H, Nishizawa M 2001 Induction of cellular antioxidative stress genes through heterodimeric transcription factor Nrf2/small Maf by antirheumatic gold(I) compounds. J Biol Chem 276:34074-34081

15. Itoh K, Chiba T, Takahashi S, Ishii T, Igarashi K, Katoh Y, Oyake T, Hayashi N, Satoh K, Hatayama I, Yamamoto M, Nabeshima Y 1997 An Nrf2/small Maf heterodimer mediates the induction of phase II detoxifying enzyme genes through antioxidant response elements. Biochem Biophys Res Commun 236:313-322

16. Alam J, Stewart D, Touchard C, Boinapally S, Choi AM, Cook JL 1999 Nrf2, a Cap'n'Collar transcription factor, regulates induction of the heme oxygenase-1 gene. J Biol Chem 274:26071-26078

17. Sun J, Hoshino H, Takaku K, Nakajima O, Muto A, Suzuki H, Tashiro S, Takahashi S, Shibahara S, Alam J, Taketo MM, Yamamoto M, Igarashi K 2002 Hemoprotein Bach1 regulates enhancer availability of heme oxygenase-1 gene. EMBO J 21:5216-5224

18. Sun J, Brand M, Zenke Y, Tashiro S, Groudine M, Igarashi K 2004 Heme regulates the dynamic exchange of Bach1 and NF-E2-related factors in the Maf transcription factor network. Proc Natl Acad Sci USA 101:1461-1466

19. Zenke-Kawasaki Y, Dohi Y, Katoh Y, Ikura T, Ikura M, Asahara T, Tokunaga F, Iwai K, Igarashi K 2007 Heme induces ubiquitination and degradation of the transcription factor bach1. Mol Cell Biol 27:6962-6971

20. Suzuki H, Tashiro S, Hira S, Sun J, Yamazaki C, Zenke Y, Ikeda-Saito M, Yoshida M, Igarashi K 2004 Heme regulates gene expression by triggering Crm1-dependent nuclear export of Bach1. EMBO J 23:2544-2553

21. Reichard JF, Motz GT, Puga A 2007 Heme oxygenase- 1 induction by NRF2 requires inactivation of the transcriptional repressor BACH1. Nucleic Acids Res 35:70747086

22. Abate A, Zhao H, Wong RJ, Stevenson DK 2007 The role of Bach1 in the induction of heme oxygenase by tin mesoporphyrin. Biochem Biophys Res Commun 354:757763

23. Kitamuro T, Takahashi K, Ogawa K, Udono-Fujimori R, Takeda K, Furuyama K, Nakayama M, Sun J, Fujita H, Hida W, Hattori T, Shirato K, Igarashi K, Shibahara S 2003 Bach1 functions as a hypoxia-inducible repressor for the heme oxygenase-1 gene in human cells. J Biol Chem 278:9125-9133

24. Ishikawa M, Numazawa S, Yoshida T 2005 Redox regulation of the transcriptional repressor Bach1. Free Radic Biol Med 38:1344-1352

25. Dennery PA, Rodgers PA, Lum MA, Jennings BC, Shokoohi V 1996 Hyperoxic regulation of lung heme oxygenase in neonatal rats. Pediatr Res 40:815-821

26. Choi AM, Sylvester S, Otterbein L, Holbrook NJ 1995 Molecular responses to hyperoxia in vivo: relationship to increased tolerance in aged rats. Am J Respir Cell Mol Biol 13:74-82

27. Yang G, Madan A, Dennery PA 2000 Maturational differences in hyperoxic AP-1 activation in rat lung. Am J Physiol Lung Cell Mol Physiol 278:L393-L398

28. Zhang W, Feng JQ, Harris SE, Contag PR, Stevenson DK, Contag CH 2001 Rapid in vivo functional analysis of transgenes in mice using whole body imaging of luciferase expression. Transgenic Res 10:423-434

29. Dignam JD, Lebovitz RM, Roeder RG 1983 Accurate transcription initiation by RNA polymerase II in a soluble extract from isolated mammalian nuclei. Nucleic Acids Res 11:1475-1489

30. Zeng PY, Vakoc CR, Chen ZC, Blobel GA, Berger SL 2006 In vivo dual crosslinking for identification of indirect DNA-associated proteins by chromatin immunoprecipitation. Biotechniques 41:694-696

31. Dennery PA, Lee CS, Ford BS, Weng YH, Yang G, Rodgers PA 2003 Developmental expression of heme oxygenase in the rat lung. Pediatr Res 53:42-47

32. Siner JM, Jiang G, Cohen ZI, Shan P, Zhang X, Lee CG, Elias JA, Lee PJ 2007 VEGF-induced heme oxygenase-1 confers cytoprotection from lethal hyperoxia in vivo. FASEB J 21:1422-1432

33. Dennery PA, Rodgers PA 1996 Ontogeny and developmental regulation of heme oxygenase. J Perinatol 16:S79-S83

34. Prows DR, Hafertepen AP, Winterberg AV, Gibbons WJ Jr, Liu C, Nick TG 2007 Genetic analysis of hyperoxic acute lung injury survival in reciprocal intercross mice. Physiol Genomics 30:271-281

35. Prows DR, Hafertepen AP, Gibbons WJ Jr, Winterberg AV, Nick TG 2007 A genetic mouse model to investigate hyperoxic acute lung injury survival. Physiol Genomics 30:262-270

36. Clerch LB, Massaro D 1992 Rat lung antioxidant enzymes: differences in perinatal gene expression and regulation. Am J Physiol 263:L466-L470

37. Clerch LB, Massaro D 1993 Tolerance of rats to hyperoxia. Lung antioxidant enzyme gene expression. J Clin Invest 91:499-508

38. Zhao H, Wong RJ, Nguyen X, Kalish F, Mizobuchi M, Vreman HJ, Stevenson DK, Contag $\mathrm{CH} 2006$ Expression and regulation of heme oxygenase isozymes in the developing mouse cortex. Pediatr Res 60:518-523

39. Zhang J, Ohta T, Maruyama A, Hosoya T, Nishikawa K, Maher JM, Shibahara S, Itoh K, Yamamoto M 2006 BRG1 interacts with Nrf2 to selectively mediate HO-1 induction in response to oxidative stress. Mol Cell Biol 26:7942-7952 